\title{
Ultrasonography of uterine leiomyomas
}

\author{
Andrzej Woźniak, Sławomir Woźniak \\ $3^{\text {rd }}$ Chair and Department of Gynaecology, Medical University, Lublin, Poland
}

\begin{abstract}
Uterine leiomyomas or uterine fibroids are the most common gynaecological tumours and occur in about 20-50\% of women around the world. Ultrasonography (USG) is the first-line imaging examination in suspected fibroids and shows high sensitivity and specificity in diagnosing this condition. Ultrasound scans can be performed transvaginally (transvaginal scan - TVS) or transabdominally (transabdominal scan - TAS); both scans have advantages and limitations, but, in general, transvaginal sonography is superior to transabdominal sonography in most cases of pelvic pathology. Whether a leiomyoma is symptomatic or not depends primarily on its size and location. During ultrasound examination, leiomyomas usually appear as well-defined, solid, concentric, hypoechoic masses that cause a variable amount of acoustic shadowing. During the examination of leiomyomas differential diagnosis is important. Some of the most common misdiagnosed pathologies are adenomyosis, solid tumours of adnexa, and endometrial polyps. Misdiagnosis of a leiomyosarcoma has the most negative consequences, presenting symptoms are very similar to benign leiomyoma, and there is no pelvic imaging technique that can reliably differentiate between those pathologies. Magnetic resonance and computer tomography might be helpful in the diagnostics of uterine leiomyoma; however, ultrasound examination is the basic imaging test confirming the existence of leiomyomas, allowing the differentiation of myomas with adenomyosis, endometrial polyps, ovarian tumours, and pregnant uterus.
\end{abstract}

Key words: ultrasound, leiomyomas, fibroids.

Uterine leiomyomas or uterine fibroids are the most common gynaecological tumours and occur in about $20-50 \%$ of women around the world, with the highest frequency in groups of black women of reproductive age. As tumours responsive to hormones are rare in prepubertal age, they accelerate in growth during pregnancy and involute with the onset of menopause. Leiomyomas are the most frequent benign tumours, with an estimated $0.1-0.8 \%$ risk of malignant transformation into sarcomas. However malignant leiomyosarcomas are rare and can arise de novo, without leiomyoma as a "base". In the human body, they are the most common tumour of all pelvic organs. Histologically leiomyomas arise from the overgrowing of the smooth muscle and connective tissue during monoclonal cell proliferation $[1,2]$.

Ultrasonography (USG) is the first-line imaging examination in the suspicion of fibroids, as a high sensitivity and specificity test. Ultrasound scans can be performed transvaginally (transvaginal scan - TVS) or transabdominally (transabdominal scan - TAS); both scans have advantages and limitations, but, in general, transvaginal sonography is superior to transabdominal sonography in most cases of pelvic pathology. TVS is definitely more sensitive in the detection of small leio- myomas and is more useful in cases of retroverted and/ or retroflexed uteruses. Moreover, TVS is helpful in patients with large amounts of bowel gas, in those unable to achieve adequate bladder filling, and in obese patients, where TAS is very difficult to perform. TAS was found to be superior in the diagnosis of fundal myomas; nevertheless, TVS is helpful in further assessment of such pathologies. The biggest limitation of TVS is the shallow depth of the scan, thus large or pedunculated myomas may be out of the scan in high-frequency probes with short focal lengths. A very important fact is that both TVS as well as TAS are totally operatordependent types of examination, so their efficiency always depends on the knowledge and skills of the operator [3-6].

Whether leiomyoma is symptomatic or not, and if it is, which symptoms are present, depends primarily on its size and location (Fig. 1, 2). In general, large leiomyomas can compress the surrounding organs and tissues, for example the bowel, bladder, or pelvic ligaments, causing constipation, dysuria, or even back pain by compressing the lumbar plexus. Large leiomyomas may also be palpable through the abdominal wall. Leiomyoma classification by FIGO lists the main types of fibroids according to their localisation: intracavitary 


\begin{tabular}{|c|c|c|}
\hline & & Coagulopathy \\
\hline Polyp & & Ovulatory dysfunction \\
\hline Adenomyosis & Submucosal & Endometrial \\
\hline Leiomyoma & & \\
\hline Malignancy \& hyperplasia & Uther & $\frac{\text { latrogenic }}{\text { Not yet classified }}$ \\
\hline
\end{tabular}

\begin{tabular}{|c|c|c|c|}
\hline \multirow{11}{*}{$\begin{array}{l}\text { Leiomyoma } \\
\text { Subclassification } \\
\text { System }\end{array}$} & \multirow{3}{*}{ SM - submucosal } & 0 & \multirow{2}{*}{$\begin{array}{l}\text { Pedunculated intracavitary } \\
<50 \% \text { intramural }\end{array}$} \\
\hline & & 1 & \\
\hline & & 2 & $\geq 50 \%$ intramural \\
\hline & \multirow[t]{6}{*}{ O-Other } & 3 & Contacts endometrium; $100 \%$ intramural \\
\hline & & 4 & Intramural \\
\hline & & 5 & Subserosal $\geq 50 \%$ intramural \\
\hline & & 6 & Subserosal $<50 \%$ intramural \\
\hline & & 7 & Subserosal Pedunculated \\
\hline & & 8 & Other (specify e.g. cervical, parasitic) \\
\hline & $\begin{array}{l}\text { Hybrid leiomyomas } \\
\text { (impact both } \\
\text { endometrium } \\
\text { and serosa) }\end{array}$ & \multicolumn{2}{|c|}{$\begin{array}{l}\text { Two numbers are listed separated by hyphen. } \\
\text { By convenction, the first refers to the relation- } \\
\text { ship with the endometrium while the second } \\
\text { refers to the relationship to the serosa. One } \\
\text { example is below }\end{array}$} \\
\hline & & $2-5$ & $\begin{array}{l}\text { Submucosal and subserosal, each } \\
\text { with less than half the diameter in the } \\
\text { endometrial and peritoneal cavities, } \\
\text { respectively }\end{array}$ \\
\hline
\end{tabular}

Fig. 1. FIGO classification of fibroids [7]
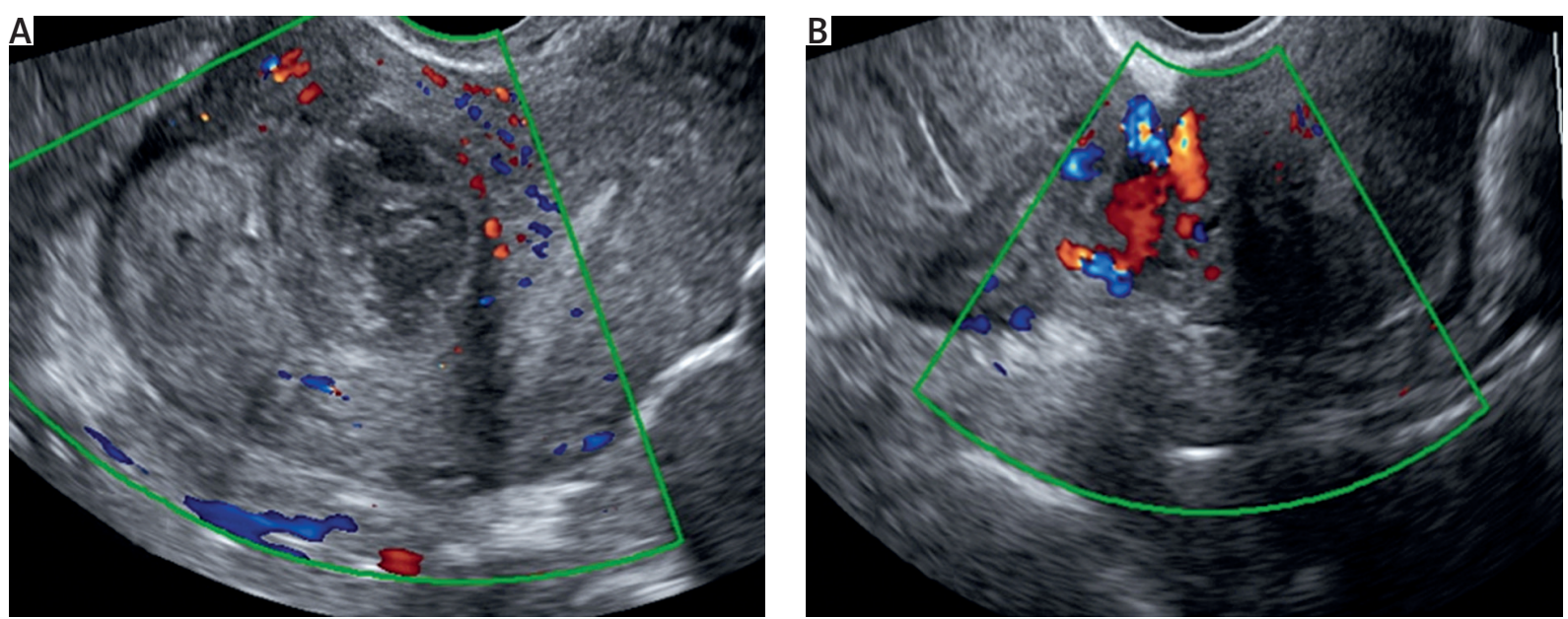

Fig. 2. A) Ultrasound image of intramural leiomyoma - FIGO-3, visible peripheral vascularisation. B) Ultrasound image of pedunculated leiomyoma - FIGO-7, visible vascularisation of the peduncle

fibroid, submucosal fibroid (least common), intramural fibroid (most common), subserosal fibroid, and pedunculated fibroid (Fig. 1) [7]. Some leiomyomas may inhibit normal vaginal deliveries, causing haemorrhage or ulceration in the case of cervical localisation. Submucosal leiomyomas may cause heavy bleeding if they protrude into the endometrial cavity and are usually the reason for long, painful menstruation, with blood clots or ab- normal uterine bleeding throughout the menstrual cycle. Abnormal bleeding can cause anaemia, headaches, general debilitation, dyspnoea, or even circulatory failure. Additionally, submucosal and intramural leiomyomas may affect fertility by inhibiting sperm transport and interfering with normal implantation. Subserosal and pedunculated leiomyomas may cause symptoms by the abovementioned pressure effect exerted on sur- 

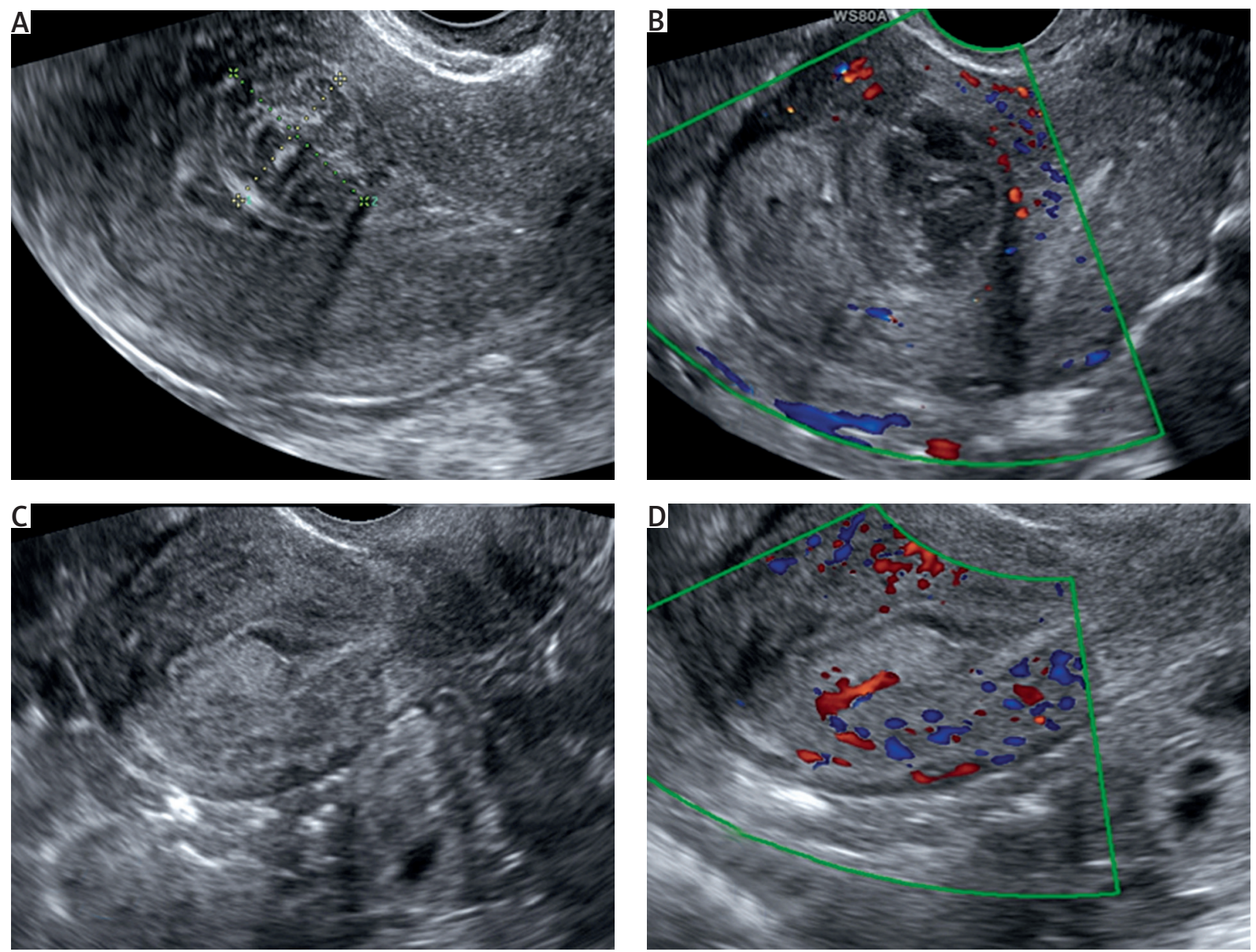

Fig. 3. Characteristic features of polyps and leiomyomas in ultrasound scans: A) heterogeneous mass in the uterine cavity fibroid, B) multiple feeding vessels, characteristic for fibroids, C) homogeneous hyperechogenic mass in the uterine cavity - endometrial polyp, D) a single feeding artery typical for polyps
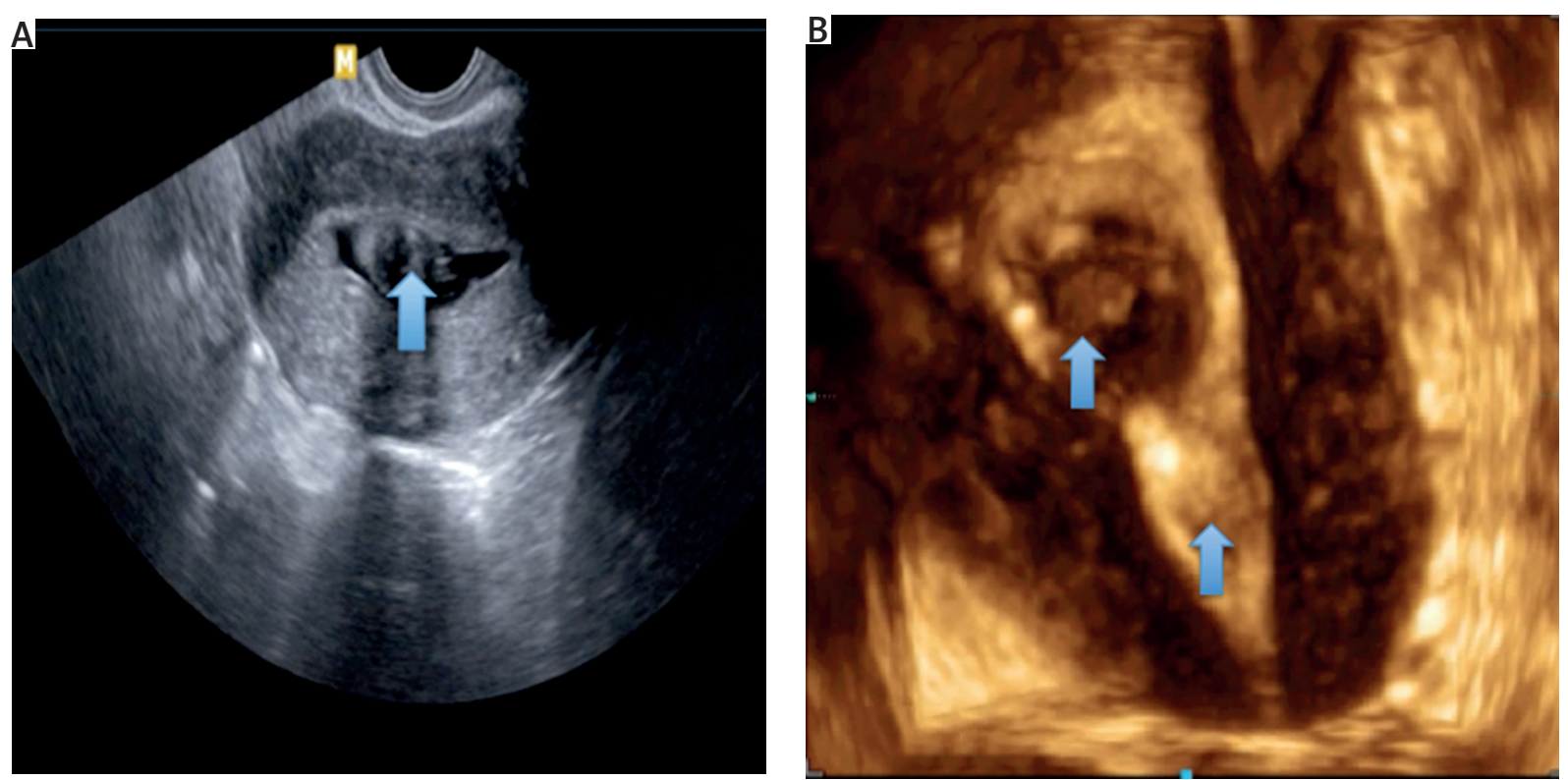

Fig. 4. Sonohysterographic images of the submucosal fibroids. A) 2-D ultrasound scan showing a uterus with a small submucosal fibroid emanating from the anterior wall (arrow). B) 3-D - two fibroids emerging from posterior and anterior wall of uterus (arrows) 
rounding tissues and may also be the reason for acute pelvic pain if it comes to necrosis or torsion of previously asymptomatic leiomyoma by torsion of the peduncle. Leiomyomas may also appear outside the uterus in extrauterine sites such as: ligaments of the pelvis, fallopian tubule, cervix, or vagina. Any leiomyoma may undergo internal haemorrhage, fibrosis, calcification, atrophy, or several types of degeneration [8-10].

The diagnosis of leiomyomas since the 1970s has been based on ultrasonography, and since the 1980s when transvaginal scans were introduced, this became a gold standard. Nowadays ultrasonography is the firstline imaging modality in the detection and evaluation of uterine leiomyomas. During ultrasound examination leiomyomas usually appear as well-defined, solid, concentric, hypoechoic masses that cause a variable amount of acoustic shadowing. However, depending on the level of calcification or/and the amount of fibrous tissue, leiomyomas may present different echogenicity, usually hyperechogenic or isoechogenic. Calcifications are seen as echogenic foci with shadowing. Sometimes leiomyomas may have anechogenic components as a result of progressing necrosis. In some difficult cases when leiomyomas are small and isoechogenic to the myometrium, the only visible ultrasound sign may be a bulge in the uterine contour. Leiomyomas of lower uterine segments like the cervix may obstruct the uterine canal. Consequently, the accumulation of fluid in the endometrial canal might be easy to notice during the examination $[5,11]$.

During the examination of leiomyomas differential diagnosis is extremely important. Some of the most common misdiagnosed pathologies are adenomyosis, solid tumours of adnexa, and endometrial polyps.

Adenomyosis is a difficult to diagnose pathology, due to the lack of significant pathognomonic signs and clinical findings, as well as differences in the histological criteria of adenomyosis recognition. Therefore, intramural leiomyomas are often misdiagnosed as adenomyosis and vice versa. However, some ultrasound features may be helpful in establishing the proper diagnosis. The following findings are suggestive of adenomyosis: globular uterine enlargement without the presence of leiomyomata, cystic anechoic spaces or lakes in the myometrium, subendometrial echoic linear striations, uterine wall thickening, heterogeneous echo texture, obscured endometrial/myometrial border, and thickening of the transition zone [12].

Subserosal fibroids and adnexal masses are pathologies that may be very difficult to distinguish. Sometimes subserosal fibroids can be pedunculated or predominantly extra-uterine. As a consequence, on ultrasound they might look similar to ovarian tumours. Because of the large fibrous component, ovarian Brenner tumours and fibrothecomas might show a low signal on T2W scans, and sometimes the proper diagnosis is not made until surgery. Another very useful tool in the diagnosis of leiomyomas is colour Doppler ultrasonography. This technique shows circumferential vascularity, blood flow, and arterial supply of the fibroid. Nevertheless, necrotic leiomyomas or those that undergo torsion do not present any blood flow $[11,13]$.

Intrauterine benign masses, such as endometrial polyps and submucosal fibroids, are sometimes misdiagnosed, which may result in improper treatment and possible harm to the patient. Homogeneous hyperechogenic masses in the uterine cavity on ultrasound are highly suggestive of endometrial polyps, but the myometrial echogenicity of uterine fibroids may vary and be hypoechogenic, isoechogenic, hyperechogenic, or mixed, which depends on the size and nature of the fibroid. Colour Doppler may be useful in distinguishing polyps from submucosal fibroids based on the vascularity of the lesions (Fig. 3). Multiple, circular feeding vessels are characteristic for fibroids, whereas a single feeding artery can be observed in most polyps. Strain elastography complements sonography in the assessment of intrauterine lesions. Strain elastography may be used to visualise the different stiffness of endometrial polyps and submucosal leiomyomas. Additionally, hysterosonography might be an important addition to TVS in accurate delineation of submucosal and intracavitary leiomyomas (Fig. 4). For further diagnostics a 3D TVS may be combined with saline instillation into the uterine cavity to differentiate submucosal leiomyomas and endometrial polyps. Three-dimensional saline contrast sonohysterography may provide even more information in this aspect $[5,14,15]$.

The pathology in which misdiagnosis results in the most negative consequences is the previously mentioned leiomyosarcoma. This rare malignant tumour is associated with very poor prognosis for the patient. It is difficult to distinguish between benign leiomyoma and malign leiomyosarcoma because the presenting symptoms are very similar. Clinically both of them are focal masses within the uterus and both often have central necrosis. There is no pelvic imaging technique that can reliably differentiate between them because both of them may show: mixed echogenic and poor echogenic parts, central necrosis, and colour Doppler findings of irregular vessel distribution, low impedance to flow, and high peak systolic velocity. In this situation, magnetic resonance might be helpful; however, it does not provide a definite diagnosis [13, 16, 17].

In some cases, magnetic resonance imagining (MRI) provides additional information as a means of further diagnostics in patients in whom ultrasound findings are confusing. With a specificity of $100 \%$, accuracy of $97 \%$, and sensitivity in the range $86-92 \%$, it is a great ally in the diagnosis of leiomyomas. Additionally, MRI is helpful in the assessment of the anatomy of the uterus and ovaries as well as in planning of myomectomy. In T1 and 
T2 MRI scans, leiomyomas appear as areas of low or intermediate signal with sharps margins $[18,19]$.

The importance of computer tomography (CT) scanning is unfortunately limited by the similar attenuation characteristics of fibroids and healthy myometrium; therefore, some leiomyomas might be overlooked. However, due to the superior contrast differentiation of CT scanning, calcified or necrotic fibroids may be more visible than on USG or MRI. Leiomyomas might distort the normal smooth uterine contour and appear as soft tissue density lesions with central or peripheral calcification [20, 21].

Ultrasound examination is the basic imaging test confirming the existence of fibroids, allowing the differentiation of myomas with adenomyosis, polyps, ovarian tumours, and pregnant uterus.

\section{Disclosure}

\section{Authors report no conflict of interest.}

\section{References}

1. Lethaby A, Vollenhoven B. Fibroids (uterine myomatosis, leiomyomas) BMJ Clin EVID 2015: pii: 0814.

2. Peddada Sd, Laughlin SK, Miner K, et al. Growth of uterine leiomyomata among premenopausal black and white women. Proc Natl Acad Sci USA 2008; 105: 19887

3. Ultrasonography in Obstetric and Gynecology. Callen PW (ed.). 5th ed. Saunders Elsevier, Philadelphia 2007.

4. Frank ML, Schafer SD, Mollers M, et al. Importance of Tranvaginal Elas tography in the Diagnosis of Uterine Fibroids and Adenomyosis. Ultraschall Med 2016; 37: 3738

5. Rashid SQ, Chou YH, Tiu CM, et al. Ultrasonography of uterine leiomyomas. J Med Ultrasound 2016; 24: 3e12.

6. Sauerbrel EE, Nguyen KT, Nolan RL, et al. A Practical Guide to Ultrasound in Obstetrics and Gynecology. 2nd ed. Lippincott-Raven, Philadelphia 1998; 52-58.

7. Munro M, Critchley H, Fraser I. The FIGO classification of causes of abnormal uterine bleeding in the reproductive. Fertil Steril 2011; 95: 2204 2208.

8. Parker WH. Etiology, symptomatology and diagnosis of uterine myomas. Fertil Steril 2007; 87: 725-736.

9. Manuro MG, Lukes AS; Abnormal Uterine Bleeding and Underlying Hemostatic Disorders Consensus Group. Abnormal uterine bleeding and underlying hemostatic disorders: report of a consensus process. Fertil Steril 2005; 84: 1335-1337.

10.Ferrero S, Abbamonte LH, Giordano M, et al. Uterine myomas, dyspareunia and sexual function. Fertil Steril 2006; 86: 1504-1510.

11. Wilde S, Scott-Barrett S. Radiological appearances of uterine fibroids. Indian J Radiol Imaging 2009; 19: 222-231.

12. Sakhel K, Abuhamad A. Sonography of adenomyosis. J Ultrasound Med 2012; 31: 805-808.

13. Amant F, Coosemans A, Debiec-Rychter M, et al. Clinical management of uterine sarcomas. Lancet Oncol 2009; 10: 1188

14. Czuczwar P, Wozniak S, Szkodziak P, et al. Elastography improves the diagnostic accuracy of sonography in differentiating endometrial polyps and submucosal fibroids. J Ultrasound Med 2016; 35: 2389-2395.

15. Tamura-Sadamori R, Emoto M, Naganuma Y, et al. The sonohysterographic difference in submucosal uterine fibroids and endometrial polyps treated by hysteroscopic surgery. J Ultrasound Med 2007; 26: 941-946.

16. Kobayashi E, Yokoyama T, Nakagawa S, et al. Pedunculated sub-serous leiomyosarcoma mimicking ovarian cancer: case report and review of literature. Gynecol Obstet 2013; 3: 157; DOI:10.4172/2161-0932.1000157.
17. Minsart AF, Ntoutoume Sima F, Vandenhoute K, et al. Does three-dimensional power Doppler ultrasound predicts histopathological findings of uterine fibroids? A preliminary study. Ultrasound Obstet Gynecol 2013; 40: 714-720.

18. Bolan C, Caserta MP. MR imaging of atypical fibroids. Abdom Radiol (NY) 2016; 41: 2332-2349.

19. Jondal DE, Wang J, Chen J, et al. Uterine fibroids: correlation between MRI appearance and stiffness via magnetic resonance elastography. Abdom Radiol (NY) 2017; doi: 10.1007/s00261-017-1314-1.

20. Dahnert W. Dahnert's Radiology Review Manual. 4th ed. Williams \& Wilkins, Baltimore 1996; 884-885.

21. Gross BH, Moss AA, Mihara K, et al. Review: computer tomography of gynecologic diseases. AJR 1983; 141: 765-773. 